\title{
Differentiable families of traceless matrix triples
}

\author{
M. Isabel García-Planas • Tetiana \\ Klymchuk
}

Received: date / Accepted: date

\begin{abstract}
Analysis of spectra of families of sets of matrices verifying certain properties is not simple because phenomena as singularities and bifurcations appear. An excellent tool for the analysis can be making use of versal deformations because of the spectrum of the family coincides with the spectrum of its versal deformation. Disposing of a versal deformation is advantageous since any perturbation of an element can be described up to equivalence by its versal deformation, and it gives the possibility to calculate bifurcation diagrams of families of elements in general position. V.I. Arnold constructed versal deformations, of a differentiable family of square matrices under conjugation and his techniques have been generalised to different cases as to matrix pencils under the strict equivalence, for example.

In this paper, we present versal deformations of elements of the Lie algebra consisting of triples of traceless matrices to coefficients on $\mathbb{F}=\mathbb{C}$ or $\mathbb{R}$, which are simultaneously diagonalizable.

Study families of traceless matrix triples have great interest because the Lie algebra is related to gauge fields because they appear in the Lagrangian
\end{abstract}

An earlier version of this paper was presented at the Conference "Linear Algebra, Matrix Analysis and Applications. ALAMA2018", held in Sant Joan d'Alacant on May/June 2018.

M. Isabel García-Planas

Universitat Politècnica de Catalunya

Departament de Matemàtiques,

Avda. Diagonal, 647,

08028 Barcelona, SPAIN

E-mail: maria.isabel.garcia@upc.edu

T. Klymchuk

Universitat Politècnica de Catalunya

Departament de Matemàtiques,

Avda. Diagonal, 647,

08028 Barcelona, SPAIN

E-mail: tetiana.klymchuk@upc.edu 
describing the dynamics of the field, then they are associated to 1-forms that take values on a certain Lie algebra. It is also of interest to note that triples of traceless matrices have some relevance for supergravity theories. Another application is found when we must give the instanton solution of Yang-Mills field can be presented in an octonion form, and it can be represented by triples of traceless matrices.

Keywords Traceless matrix - Versal deformation - Families of sets of matrices

Mathematics Subject Classification (2010) 15A21 · 15A90

\section{Introduction}

Analysis of versal families of elements of a variety under some equivalence relation is a significant challenge both from the theoretical and practical point of view. Disposing of a versal deformation is advantageous since any perturbation of an element can be described up to equivalence by its versal deformation, and it gives the possibility to calculate bifurcation diagrams of families of elements in general position.

V.I. Arnold constructed versal deformations, of a differentiable family of square matrices under conjugation [1] and his techniques have been generalised by several authors to matrix pencils under the strict equivalence [9], pairs or triples of matrices under the action of the general linear group [12], pairs of matrices under the feedback similarity [6], for example.

In this paper, we are interested in versal deformations of elements of the Lie algebra of triples of traceless matrices to coefficients on $\mathbb{F}=\mathbb{C}$ or $\mathbb{R}$

$$
\mathfrak{M}=\left\{(A, B, C) \in \times_{3} M_{n}(\mathbb{F}) \mid \operatorname{tr} A=\operatorname{tr} B=\operatorname{tr} C=0\right\} \approx \times_{3} \mathfrak{s l}(n, \mathbb{F})
$$

of the Lie group $\mathfrak{G}=\left\{(A, B, C) \in \times M_{n}(\mathbb{F}) \mid \operatorname{det} A=\operatorname{det} B=\operatorname{det} C=1\right\} \approx$ $\times{ }_{3} S l(n, \mathbb{F})$. And particularly, we are interested for the case where the matrices in the triple are simultaneously diagonalizable. Remember that a $m$-triple of matrices $(A, B, C)$ is simultaneously diagonalizable if and only if there exist an invertible matrix $P$ such that $\left(D_{1}, D_{2}, D_{3}\right)=\left(P A P^{-1}, P B P^{-1}, P C P^{-1}\right)$ with each $D_{i}$ diagonal; and a necessary and sufficient condition for simultaneous diagonalization of the triple $\left(X_{1}, X_{2}, X_{3}\right)$ is there exist a basis $\left\{v_{1}, \ldots, v_{n}\right\}$ of $v \in$ $\mathbb{F}^{n}$ such that $v_{j} \in \cap_{i=1}^{3} \operatorname{Ker}\left(X_{i}-\lambda_{i}^{j}\right) I$, where $\lambda_{j}^{i} \in \operatorname{Spec} X_{i}=\left\{\lambda_{1}^{i}, \ldots, \lambda_{n}^{i}\right\}$. Conditions for simultaneous diagonalizable triples can be found in [8].

Study families of traceless matrices have great interest because the Lie algebra is related to gauge fields because they appear in the Lagrangian describing the dynamics of the field, then they are associated to 1-forms, (Chern-Simons terms (CS), that take values on a certain Lie algebra, [2], [7].

On a related side, it seems that a direct relevance for supergravity theories is provided by the cases $n=2$ and $\mathbb{F}=\mathbb{R}$ respectively $\mathbb{F}=\mathbb{C}$ of the treatment. The case $\mathbb{F}=\mathbb{R}$ is relevant for the so-called STU model [5], [11], of $n=2$ Maxwell-Einstein supergravity in four space-time dimensions coupled to three 
vector multiplets, in which the continuous limit of the U-duality Lie algebra is $\times_{3} \mathfrak{s l}(2, \mathbb{R})$ (one would better use the symbol $+_{3}$ for Lie algebras). The case $\mathbb{F}=\mathbb{C}$ is relevant to quantum information theory, and in particular to the entanglement of three qubits [3]; this extends, for generic $n \geq 2$, to the entanglement of three quantum n-dits. As another application, we mention the case where the instanton solution of a Yang-Mills field can be presented in an octonion form, and it can be represented by triples of traceless matrices ([10]).

Finally, we want to point out that if we restrict from triples to pairs and working on the complex numbers field $\mathbb{F}=\mathbb{C}$, the results may be relevant to a further characterization of the generalization of the Bloch-Messiah Theorem provided in [4].

The paper is organised as follows, general concepts of Lie groups actions and the versal deformation theory are given in Section 2 and 3. In section 4, the explicit miniversal deformation for triples of traceless matrices that are simultaneously diagonalizable is presented.

\section{Lie group action}

The set $\mathfrak{M}$ of triples of traceless matrices is a differentiable manifold and the classical Lie group $\mathcal{G}=\left\{P \in M_{n}(\mathbb{F}) \mid \operatorname{det} P \neq 0\right\}$ acts over $\mathfrak{M}$ in the following manner

$$
\begin{aligned}
\alpha: \mathcal{G} \times \mathfrak{M} & \longrightarrow \mathfrak{M} \\
(P,(A, B, C)) & \longrightarrow \alpha(P,(A, B, C))=\left(P^{-1} A P, P^{-1} B P, P^{-1} C P\right)
\end{aligned}
$$

Clearly $\alpha$ is differentiable and surjective.

Remark $1 \mathcal{G}_{1}=S l(n, \mathbb{F})=\{P \in G l(n, \mathbb{C}) \mid \operatorname{det} P=1\}$ acts over $\mathfrak{M}$ in the same manner.

Fixing $X_{0}=\left(A_{0}, B_{0}, C_{0}\right)$ we have the differentiable map

$$
\begin{aligned}
\alpha_{X_{0}}: & \mathcal{G} \longrightarrow \mathfrak{M} \\
P & \longrightarrow \alpha\left(P,\left(A_{0}, B_{0}, C_{0}\right)\right)=\left(P^{-1} A_{0} P, P^{-1} B_{0} P, P^{-1} C_{0} P\right)
\end{aligned}
$$

and

$\operatorname{Im} \alpha_{X_{0}}=\mathcal{O}\left(X_{0}\right)$ called the orbit of $X_{0}$

$\operatorname{Stab}\left(X_{0}\right)=\left\{P \in \mathcal{G} \mid \alpha_{X_{0}}(P)=X_{0}\right\}$ called the stabilizer of $X_{0}$

The map $\alpha_{0}$ defines an equivalence relation over $\mathfrak{M}$ which equivalent classes are the orbits of the elements.

Let $d \alpha_{X_{0}}: T_{I} \mathcal{G} \longrightarrow \mathfrak{M}$ be the differential of $\alpha_{X_{0}}$ at the unit element $I$. It is easy to compute $d \alpha_{X_{0}}(P)$ :

$$
d \alpha_{X_{0}}(P)=\left(\left[A_{0}, P\right],\left[B_{0}, P\right],\left[C_{0}, P\right]\right) \in \mathfrak{M}, \quad P \in T_{I} \mathcal{G}
$$


If we define scalar products in $\mathfrak{M}$ and $T_{I} \mathcal{G}$, we can consider the adjoint application of $d \alpha_{X_{0}}$. The Euclidean scalar products considered in this paper are defined as follows:

For all $X_{i}=\left(A_{i}, B_{i}, C_{i}\right) \in \mathfrak{M}$ and for all $P_{i} \in T_{I} \mathcal{G}$

$$
\begin{aligned}
& \left\langle X_{1}, X_{2}\right\rangle_{1}=\operatorname{trace}\left(A_{1} A_{2}^{*}\right)+\operatorname{trace}\left(B_{1} B_{2}^{*}\right)+\operatorname{trace}\left(C_{1} C_{2}^{*}\right), \\
& \left\langle P_{1}, P_{2}\right\rangle_{2}=\operatorname{trace}\left(P_{1} P_{2}^{*}\right),
\end{aligned}
$$

where $A^{*}$ denotes the conjugate transpose of a matrix $A$.

The adjoint linear mapping $d \alpha_{X_{0}}^{*}: \mathfrak{M} \longrightarrow T_{I} \mathcal{G}$ is defined by the relation

$$
\left\langle d \alpha_{X_{0}}(P), Z\right\rangle_{1}=\left\langle P, d \alpha_{x_{0}}^{*}(Z)\right\rangle_{2}, \quad P \in T_{I} \mathcal{G}, \quad Z \in \mathfrak{M} .
$$

It is straightforward to find

$$
d \alpha_{X_{0}}^{*}(W)=\left(\left[X^{*}, A_{0}\right]+\left[Y^{*}, B_{0}\right]+\left[Z^{*}, C_{0}\right]\right) \in T_{I} \mathcal{G}, \quad W=(X, Y, Z) \in \mathfrak{M}
$$

The mappings $d \alpha_{X_{0}}$ and $d \alpha_{X_{0}}^{*}$ provide a simple description of the tangent spaces $T_{X_{0}} \mathcal{O}\left(X_{0}\right), T_{I} \mathcal{S} t a b\left(X_{0}\right)$ and their normal complements $\left(T_{X_{0}} \mathcal{O}\left(X_{0}\right)\right)^{\perp}$, $\left(T_{I} \mathcal{S}\left(X_{0}\right)\right)^{\perp}$

Theorem 1 The tangent spaces to the orbit and stabilizer of the matrix pencil $X_{0}$ and the corresponding normal complementary subspaces with respect to $\mathfrak{M}$ and $T_{I} \mathcal{G}$ can be found in the following form

1. $T_{X_{0}} \mathcal{O}\left(X_{0}\right)=\operatorname{Im} d \alpha_{X_{0}} \subset \mathfrak{M}$.

2. $\left(T_{X_{0}} \mathcal{O}\left(X_{0}\right)\right)^{\perp}=\operatorname{Ker} d \alpha_{x_{0}}^{*} \subset \mathfrak{M}$,

3. $T_{I} \mathcal{S} \operatorname{tab}\left(X_{0}\right)=\operatorname{Ker} d \alpha_{X_{0}} \subset T_{I} \mathcal{G}$,

4. $\left(T_{I} \mathcal{S} \operatorname{tab}\left(X_{0}\right)\right)^{\perp}=\operatorname{Im} d \alpha_{X_{0}}^{*} \subset T_{I} \mathcal{G}$.

After this theorem, it is easy to compute these spaces.

Corollary 1 1. $T_{X_{0}} \mathcal{O}\left(X_{0}\right)=\left\{\left(\left[P, A_{0}\right],\left[P, B_{0}\right],\left[P, C_{0}\right]\right) \mid P \in T_{I} \mathcal{G}\right\}$

2. $\left(T_{X_{0}}\left(\mathcal{O}\left(X_{0}\right)\right)^{\perp}=\left\{(X, Y, Z) \in \mathfrak{M} \mid\left[X^{*}, A_{0}\right]+\left[Y^{*}, B_{0}\right]+\left[Z^{*}, C_{0}\right]=0\right\}\right.$

3. $T_{I} \operatorname{Stab}\left(X_{0}\right)=\left\{P \in M_{n}(\mathbb{F}) \mid\left[A_{0}, P\right]=0,\left[B_{0}, P\right]=0,\left[C_{0}, P\right]=0\right\}$

4. $\left(T_{I} \mathcal{S} \operatorname{tab}\left(X_{0}\right)\right)^{\perp}=\left\{\left[X^{*}, A_{0}\right]+\left[Y^{*}, A_{0}\right]+\left[Z^{*}, A_{0}\right] \mid \forall(X, Y, Z) \in \mathfrak{M}\right\}$

Remark 2 Note that, the tangent space to the stabilizer of a triple $T_{I} \mathcal{S} t a b\left(X_{0}\right)$ coincides with the centralizer of this element $C_{X_{0}}$ defined as $C_{X_{0}}=\{Z \in$ $\left.M_{n}(\mathbb{F}) \mid A_{0} Z-Z A_{0}=0, B_{0} Z-Z B_{0}=0, C_{0} Z-Z C_{0}=0\right\}$.

Moreover, $C_{X_{0}}=C_{A_{0}} \cap C_{B_{0}} \cap C_{C_{0}}$ where $C_{A_{0}}, C_{B_{0}}$ and $C_{C_{0}}$ are the centralizers of $A_{0}, B_{0}, C_{0}$ under similarity that also correspond to the tangent spaces of the stabilizers of $A_{0}, B_{0}, C_{0}$ respectively, with respect the similarity action of $\mathcal{G}$ over the space of square matrices. So,

$$
T_{I} \mathcal{S} \operatorname{tab}\left(X_{0}\right)=T_{I} \mathcal{S} t a b\left(A_{0}\right) \cap T_{I} \mathcal{S} t a b\left(B_{0}\right) \cap T_{I} \mathcal{S t a b}\left(C_{0}\right)
$$




\section{Versal deformations}

Definition 1 A deformation of an element $X_{0} \in \mathfrak{M}$ is a family of elements of $\mathfrak{M}$ indexed by $\lambda \in \Lambda \varphi: \Lambda \longrightarrow \mathfrak{M}$ where $\Lambda \subset \mathbb{F}^{m}$ is a neighborhood of 0 , and where $\varphi(0)=X_{0}$ and $\varphi$ depends holomorphically (smoothly) on the parameters.

Definition 2 A deformation $\varphi(\lambda)=\varphi\left(\lambda_{1}, \ldots, \lambda_{m}\right)$ of $X_{0}$ is versal if and only if for any deformation $\varphi^{\prime}\left(\mu_{1}, \ldots, \varphi_{k}\right) \in \mathfrak{M}$ of $X_{0}, \varphi^{\prime}(\mu)$ is induced by $\varphi(\lambda)$, i.e., there exists a neighborhood $V$ of 0 in $\mathbb{F}^{k}$, a map $\psi: V \longrightarrow \mathbb{F}^{m}$ with $\psi(0)=0$, and a map $g: V \longrightarrow G$ with $g(0)=I$ such that $\forall \mu \in V$, $\varphi^{\prime}(\mu)=g(\mu) \varphi(\psi(\mu)) g^{-1}(\mu)$ with $\psi$ and $g$ holomorphic (smooth).

It is obvious that if we have a versal deformation of an element automatically we have a versal deformation of any element that is equivalent to it, since if $X=\alpha\left(g, X_{0}\right)$ is an equivalent element of $X_{0}$ and $\varphi(\lambda)$ is a versal deformation of $X^{\prime}$ then $\alpha\left(g^{-1}, X(\lambda)\right)$ is a versal deformation of $X_{0}$.

A versal deformation having minimal number of parameters is called miniversal.

The following result was proved by Arnold [1], in the case where $\operatorname{Gl}(n ; \mathbb{C})$ acts on $M_{n}(\mathbb{C})$, and was generalized by Tannenbaum [12], in the case where a Lie group acts on a complex manifold. It provides the relationship between a versal deformation of $X_{0}$ and the local structure of the orbit.

\section{Theorem 2 ([12])}

1. A deformation $\varphi(\lambda)$ of $\left(X_{0}\right)$ is versal if and only if it is transversal to the orbit $\mathcal{O}\left(X_{0}\right)$ at $\left(X_{0}\right)$.

2. Minimal number of parameters of a versal deformation is equal to the codimension of the orbit of $X_{0}$ in $\mathfrak{M}, \ell=\operatorname{codim} \mathcal{O}\left(X_{0}\right)$.

Corollary 2 Then $\varphi(\lambda)=X_{0}+\left(T_{X_{0}} \mathcal{O}\left(X_{0}\right)\right)^{\perp}$ for some scalar product is a miniversal deformation.

Let $\left\{v_{1}, \ldots, v_{\ell}\right\}$ be a basis of any arbitrary complementary subspace $\left(T_{X_{0}} \mathcal{O}\left(X_{0}\right)^{c}\right.$ to $T_{X_{0}} \mathcal{O}\left(X_{0}\right)$.

\section{Corollary 3 The deformation}

$$
\varphi: \Lambda \subset \mathbb{C}^{\ell} \longrightarrow M, \quad \varphi(\lambda)=x_{0}+\sum_{i=1}^{\ell} \lambda_{i} v_{i}
$$

is a miniversal deformation.

The following proposition relates the orthogonal space to the orbit of a given matrix triple at this element, with the orthogonal space to the orbit of an equivalent matrix triple at this equivalent element. 
Proposition 1 Let $\left(A_{0}, B_{0}, C_{0}\right)$ and $\left(A_{1}, B_{1}, C_{1}\right)$ be two equivalent matrix triples. Then,

$$
\left(X_{0}, Y_{0}, Z_{0}\right) \in T_{\left(A_{0}, B_{0}, C_{0}\right)} \mathcal{O}\left(A_{0}, B_{0}, C_{0}\right)^{\perp}
$$

if and only if

$$
\left(X_{1}, Y_{1}, Z_{1}\right) \in T_{\left(A_{1}, B_{1}, C_{1}\right)} \mathcal{O}\left(A_{1}, B_{1}, C_{1}\right)^{\perp}
$$

with $X_{1}=P^{*} X_{0} P^{-*}, Y_{1}=P^{*} Y_{0} P^{-*}$, and $Z_{1}=P^{*} Z_{0} P^{-*}$ in which $P^{-*}:=$ $\left(P^{-1}\right)^{*}$ and $P$ such that $A_{0}=P A_{1} P^{-1}, B_{0}=P B_{1} P^{-1}$ and $C_{0}=P C_{1} P^{-1}$.

Proof $\left(A_{0}, B_{0}, C_{0}\right)$ and $\left.\left(A_{1}, B_{1}, C_{1}\right)\right)$ are two elements in the same orbit that means that $A_{0}=P A_{1} P^{-1}, B_{0}=P B_{1} P^{-1}$ and $C_{0}=P C_{1} P^{-1}$. Moreover, $\left(X_{0}, Y_{0}, Z_{0}\right) \in T_{\left(A_{0}, B_{0}, C_{0}\right)} \mathcal{O}\left(A_{0}, B_{0}, C_{0}\right)^{\perp}$ if and only if

$$
\begin{aligned}
& X_{0}^{*} P A_{1} P^{-1}-P A_{1} P^{-1} X_{0}^{*}+Y_{0}^{*} P B_{1} P^{-1}-P B_{1} P^{-1} Y_{0}^{*}+Z_{0}^{*} P C_{1} P^{-1}- \\
& P C_{1} P^{-1} Z_{0}^{*}=0 .
\end{aligned}
$$

then:

$$
\begin{aligned}
& P^{-1} X_{0}^{*} P A_{1}-A_{1} P^{-1} X_{0}^{*} P+P^{-1} Y_{0}^{*} P B_{1}-B_{1} P^{-1} Y_{0}^{*} P+P^{-1} Z_{0}^{*} P C_{1}- \\
& C_{1} P^{-1} Z_{0}^{*} P^{-1}=0 .
\end{aligned}
$$

That is to say

$$
\begin{aligned}
& \left(P^{*} X_{0} P^{-*}, P^{*} Y_{0} P^{-*}, P^{*} Z_{0} P^{-*}\right)= \\
& \left(X_{1}, Y_{1}, Z_{1}\right) \in\left(T_{\left(A_{1}, B_{1}, C_{1}\right)} \mathcal{O}\left(A_{1}, B_{1}, C_{1}\right)\right)^{\perp} .
\end{aligned}
$$

\section{Explicit miniversal deformation}

We are interested with miniversal deformation of the elements in $\mathcal{S}=\{X \in$ $\mathcal{M} \mid \exists P \in G$ such that $\left.P^{-1} A P=D_{1}, P^{-1} B P=D_{2}, P^{-1} C P=D_{3}\right\} \subset \mathcal{M}$.

Taking into account proposition 1 , we can consider the element in a reduced form (all matrices in its diagonal form)

$$
D_{1}=\operatorname{diag}\left(\lambda_{11}, \ldots, \lambda_{1 n}\right), D_{2}=\operatorname{diag}\left(\lambda_{21}, \ldots, \lambda_{2 n}\right), D_{3}=\operatorname{diag}\left(\lambda_{31}, \ldots, \lambda_{3 n}\right)
$$

It is not a restriction because of

First case.

Suppose that $\lambda_{i j} \neq \lambda_{i k}$, for all $i, j, k=1,2,3$. Applying Corollary 3 of section 3 and Corollary 1 item 2 of section 2 and solving the corresponding system, we have that, a general element $(X, Y, Z)$ of $\left(T_{X_{0}} \mathcal{O}\left(X_{0}\right)\right)^{\perp}$ is given by

$$
\left(\left(\begin{array}{cccc}
x_{11} & x_{12} & \ldots & x_{1 n} \\
x_{21} & x_{22} & \ldots & x_{2 n} \\
\vdots & \vdots & & \vdots \\
x_{n 1} & x_{n 2} & \ldots & -\sum_{i=1}^{n-1} x_{i i}
\end{array}\right),\left(\begin{array}{cccc}
y_{11} & y_{12} & \ldots & y_{1 n} \\
y_{21} & y_{22} & \ldots & y_{2 n} \\
\vdots & \vdots & & \vdots \\
y_{n 1} & y_{n 2} & \ldots & -\sum_{i=1}^{n-1} y_{i i}
\end{array}\right)\right.
$$




$$
\left.\left(\begin{array}{cccc}
z_{11} & -\frac{\lambda_{11}-\lambda_{12}}{\lambda_{31}-\lambda_{32}} x_{12}-\frac{\lambda_{21}-\lambda_{22}}{\lambda_{31}-\lambda_{32}} y_{12} & \ldots-\frac{\lambda_{11}-\lambda_{1 n}}{\lambda_{31}-\lambda_{3 n}} x_{1 n}-\frac{\lambda_{21}-\lambda_{2 n}}{\lambda_{31}-\lambda_{3 n}} y_{1 n} \\
-\frac{\lambda_{11}-\lambda_{12}}{\lambda_{31}-\lambda_{32}} x_{21}-\frac{\lambda_{21}-\lambda_{22}}{\lambda_{31}-\lambda_{32}} y_{21} & z_{22} & \ldots-\frac{\lambda_{12}-\lambda_{1 n}}{\lambda_{32}-\lambda_{3 n}} x_{2 n}-\frac{\lambda_{22}-\lambda_{2 n}}{\lambda_{32}-\lambda_{3 n}} y_{2 n} \\
\vdots & \vdots & \vdots \\
-\frac{\lambda_{11}-\lambda_{1 n}}{\lambda_{31}-\lambda_{3 n}} x_{n 1}-\frac{\lambda_{21}-\lambda_{n 2}}{\lambda_{31}-\lambda_{3 n}} y_{n 1} & -\frac{\lambda_{12}-\lambda_{1 n}}{\lambda_{32}-\lambda_{3 n}} x_{n 2}-\frac{\lambda_{22}-\lambda_{2 n}}{\lambda_{32}-\lambda_{3 n}} y_{n 2} & \ldots & -\sum_{i=1}^{n-1} z_{i i}
\end{array}\right)\right)
$$

The number of parameters of a miniversal deformation is $\operatorname{dim}\left(T_{X_{0}} \mathcal{O}\left(X_{0}\right)\right)^{\perp}=$ $2 n^{2}+n-3$

So,

$$
\begin{aligned}
\varphi: \Lambda \subset \mathbb{F}^{2 n^{2}+n-3} & \longrightarrow \mathfrak{M} \\
\lambda & \longrightarrow\left(D_{1}, D_{2}, D_{3}\right)+\{(X, Y, Z)\},
\end{aligned}
$$

is a miniversal deformation of $\left(D_{1}, D_{2}, D_{3}\right)$.

Notice that

$$
\begin{aligned}
\varphi_{1}: \Lambda_{1} \subset \mathbb{F}^{n^{2}-1} & \longrightarrow M_{n}(\mathbb{C}) \\
\lambda_{1} & \longrightarrow D_{1}+\{X\} \\
\varphi_{2}: \Lambda_{2} \subset \mathbb{F}^{n^{2}-1} & \longrightarrow M_{n}(\mathbb{C}) \\
\lambda_{2} & \longrightarrow D_{2}+\{Y\} \\
\varphi_{3}: \Lambda_{3} \subset \mathbb{F}^{n-1} & \longrightarrow M_{n}(\mathbb{C}) \\
\lambda_{3} & \longrightarrow D_{3}+\{Z\}
\end{aligned}
$$

with $\Lambda_{1} \times \Lambda_{2} \times \Lambda_{3}=\Lambda$ and $\left(\lambda_{1}, \lambda_{2} \Lambda_{3}\right)=\lambda$, are versal deformations of $D_{1}, D_{2}$ and $D_{3}$ respectively, but not miniversal deformations.

Example 1 i) A miniversal deformation of

$$
\left(\left(\begin{array}{ll}
1 & 0 \\
0 & 2
\end{array}\right),\left(\begin{array}{ll}
3 & 0 \\
0 & 4
\end{array}\right),\left(\begin{array}{ll}
5 & 0 \\
0 & 6
\end{array}\right)\right)
$$

is the following

$$
\left(\left(\begin{array}{cc}
1+x_{11} & x_{12} \\
x_{21} & 2+x_{22}
\end{array}\right),\left(\begin{array}{cc}
3+y_{11} & y_{12} \\
y_{21} & 4+y_{22}
\end{array}\right),\left(\begin{array}{cc}
5+z_{11} & -x_{12}-y_{12} \\
-x_{21}-y_{21} & 6+z_{22}
\end{array}\right)\right)
$$

Restricted to the diagonal triples we have:

$$
\left(\left(\begin{array}{ll}
1+x_{11} & \\
& 2+x_{22}
\end{array}\right),\left(\begin{array}{ll}
3+y_{11} & \\
& 4+y_{22}
\end{array}\right),\left(\begin{array}{ll}
5+z_{11} & \\
& 6+z_{22}
\end{array}\right)\right)
$$

ii) A miniversal deformation of

$$
\left(\left(\begin{array}{cc}
1 & 0 \\
0 & -1
\end{array}\right),\left(\begin{array}{cc}
2 & 0 \\
0 & -2
\end{array}\right),\left(\begin{array}{cc}
3 & 0 \\
0 & -3
\end{array}\right)\right)
$$

is the following

$$
\left(\left(\begin{array}{cc}
1+x_{11} & x_{12} \\
x_{21} & -1-x_{11}
\end{array}\right),\left(\begin{array}{cc}
2+y_{11} & y_{12} \\
y_{21} & -2-y_{11}
\end{array}\right),\left(\begin{array}{cc}
3+z_{11} & -\frac{1}{3} x_{12}-\frac{2}{3} y_{12} \\
-\frac{1}{3} x_{21}-\frac{2}{3} y_{21} & -3-z_{11}
\end{array}\right)\right) .
$$

Restricted to the diagonal triples we have:

$$
\left(\left({ }^{1+x_{11}}-1-x_{11}\right),\left({ }^{2+y_{11}}-2-y_{11}\right),\left({ }^{3+z_{11}}-3-z_{11}\right)\right) \text {. }
$$


Note that the triple of the second example, unlike the first, is traceless, this has led to a reduction in the number of parameters and therefore of the possibilities of being disturbed by maintaining the diagonal character and the traceless of the matrices.

\section{References}

1. V. I. Arnold. (1971). On matrices depending on parameters, Russian Math. Surveys, 26:2, pp.29-43.

2. Bonora, L., Cvitan, M., Dominis Prester, P., Pallua, S., Smolić, I. 2011. Gravitational Chern-Simons Lagrangian terms and spherically symmetric spacetimes. Classical and Quantum Gravity, 28 19, pp.195009.

3. Borsten, L., Dahanayake, D., Duff, M.J., Rubens, W., Ebrahim, H. Freudenthal triple classification of three-qubit entanglement, Phys. Rev. A80 (2009) 032326.

4. Dobaczewski, J. Generalization of the Bloch-Messiah-Zumino theorem, Phys. Rev. C62, 017301 (2000).

5. Duff, M.J., Liu,J.T, Rahmfeld, J. Four-dimensional string-string-string triality, Nucl. Phys. B459 (1996) 125.

6. J. Ferrer, M. I. Garcia-Planas, and F. Puerta, Brunowsky local form of a holomorphic family of pairs of matrices, Linear Algebra Appl., 253 (1997), pp. 175-198.

7. Fré, P., Giambrone, A., Grassi, P.A., Vasko, P. (2019). The $\mathcal{N}_{3}=3 \rightarrow \mathcal{N}_{3}=4$ enhancement of Super Chern-Simons theories in $D=3$, Calabi HyperKähler metrics and M2-branes on the $\mathcal{C}\left(N^{0,1,0}\right)$ conifold. arXiv:1906.11672.

8. Garcia-Planas, M.I. On simultaneously and approximately simultaneously diagonalizable m- tuples of matrices, Fund. J. Math. Appl. 2 (1), (2019), 50-55.

9. Garcia-Planas, M.I., V. V. Sergeichuk, V.V. Simplest miniversal deformations of matrices, matrix pencils, and contragredient matrix pencils, Linear Algebra Appl., 302-303 (1999), pp.45-61.

10. Okubo, S. Introduction to Octonion and Other Non-Associative Algebras in Physics. Cambridge University Press. (1995)

11. Sen, A., Vafa, C. Dual pairs of type II string compactification, Nucl.Phys. B455 (1995), pp. 165-187.

12. A. Tannenbaum. (1981). Invariance and System Theory: Algebraic and geometric Aspects, Lecture Notes in Math. 845, Springer-Verlag. 